\title{
EIN 3-ACHSIGER SIMULATOR ZUR VERSCHLEISSUNTERSUCHUNG VON HÜFTENDOPROTHESEN UNTER REALEN RANDBEDINGUNGEN
}

\author{
Z. Wendt, U. Boenick \\ Institut für Mikrotechnik und Medizintechnik, Technische Universität Berlin \\ zongwen@bmt1.kf.tu-berlin.de
}

\section{EINLEITUNG}

Ein entscheidendes Qualitätsmerkmal in der Hüftendoprothetik ist die Langzeitverträglichkeit der Implantate. Diese Bestrebung wird jedoch durch die nicht selten vorkommenden Osteolysenerscheinungen gefährdet, deren Grund oft auf die Abriebpartikeln der artikulierenenden Gelenkpartner zurückzuführen sind. Da der Verschleißmechanismus aufgrund individuell vielfältiger Randbedingungen sehr komplex sein könnte, sind bei der ständigen Werkstoffverbesserung die in vivo und in vitro Verschleißuntersuchungen von großer Bedeutung. Im Gegensatz zu klinischen Beobachtungen liefern Simulatortests realitätsnahe Auskünfte unter kontrollierbaren Randbedingungen innerhalb kürzester Zeit. Dieser Beitrag stellt einen neuentwickelten dreiachsigen Hüftgelenksimulator zur Verschleißuntersuchung vor. Der Simulator ist so konzipiert, daß die natürlichen Gegebenheiten hinsichtlich Kinematik, Testumgebung und anatomischer Lage möglichst genau nachgebildet werden. Ein Verschleißtest nach ISO/DIS-14242 sowie unter besonderen Testbedingungen läßt sich mit diesem Simulator durchfuhren.

\section{MATERIALIEN UND METHODEN}

Gegenstand der Untersuchung ist vorwiegend der Abrieb durch das Artikulieren des Femurkopfes gegen die Azetabulumpfanne nach einer vorgegebenen Zyklenanzahl unter definierter Belastung. Das Rotieren des Femurkopfes in der Pfanne läßt sich auf die sagittale, die frontale und die horizontale Ebene zerlegen und ergibt dadurch eine Extensions-/Flexionsbewegung, eine Abduktions-/Adduktionsbewegung und eine Innen/Außenrotationsbewegung. Der Simulator führt alle drei Winkelbewegungen aus.

Abb.1 zeigt einen schematischen Aufbau und Abb. 2 das Antriebsprinzip des hier vorgestellten Simulators. $\mathrm{Er}$ besteht im wesentlichen aus einer geschlossenen Testprothesenkammer (1) und einer geschlossenen Referenzprothesenkammer (2), Antriebselementen $(4,5,6)$ für die Realisierung der Kinematik, einer krafterzeugenden Komponente (3), sowie Meßsensoren. In den Test- und Referenzprothesenkammern, die sich seriell auf der Kraftwirkungslinie befinden, sind zwei identische Gleitpartner eingebaut, die von einer temperierten synthetisierten Gelenkflüssigkeit durchströmt werden. Beide Gleitpartner werden identisch belastet, wobei nur die Femur- und die Azetabulumkomponente in der Testprothesenkammer gegeneinander artikulieren. Die Referenzprothese dient allein zur Kompensation der Fehleranteile durch die Wasseraufnahme und das Kriechen der Kunststoffpfanne während des Tests. Wie in der Wirklichkeit auch der Fall ist, gehen alle Winkelbewegungen von den Femurkomponenten aus, wobei die beiden Schwenkbewegungen mit Hilfe von Hydraulikzylindern $(5,6)$ und die Innen-/Außenrotation mit einem Schrittmotor (4) realisiert werden. Damit auf die beiden Prothesen auch die gleichen Gelenkkräfte wirken, nimmt eine Federkombination die Gewichte der Konstruktionsteile auf. Eine Selbstzentrierung befindet sich oberhalb der Testpfanne und erlaubt, wie in den realen Gegebenheiten, eine weitere Zentrierung der verformten Pfanne um das Rotationszentrum. Sämtliche Regelungsgvorgänge erfolgen digital in Echtzeit. Alle Steuerparameter sowie Kraft- und Winkelabläufe lassen sich bequem online einstellen.
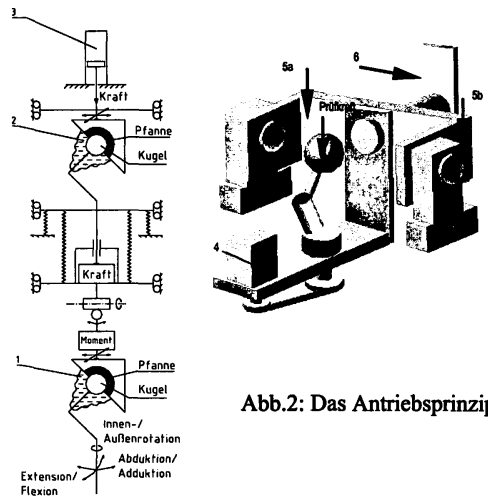

Abb.2: Das Antriebsprinzip

Abb.1: Schematischer Aufbau

des Hüftgelenksimulators

\section{LITERATURHINWEISE}

[1] E.W. Morscher, Endoprothetik, Springer, Heidelberg, Berlin 1995 